\title{
ON ASYMPTOTICS OF FUNCTIONALS OF RANDOM FIELDS WITH LONG-RANGE DEPENDENCE
}

\author{
TAREQ ALODAT
}

(Received 13 January 2020; first published online 20 February 2020)

2010 Mathematics subject classification: primary 60G60; secondary 60F17, 62M30, 62M40.

Keywords and phrases: long-range dependence, Hermite distributions, additive functionals, filtered random fields, self-similar processes.

The assumption of independence in various statistical models is only an approximation as it is often not valid for various real data. In many real applications, researchers have observed that data show strong dependencies and large correlations even between distant observations.

In probability and statistical applications, methods to deal with random variables differ according to their dependence structures. The covariance and spectral functions are commonly used to characterise the dependence properties of random fields and processes. Random fields and processes are classified according to their type of dependence into two types. The first type is random fields with weak dependence (or short memory). A random field is weakly dependent if its covariance function is integrable. These fields have bounded spectral density functions and their covariance functions are rapidly decaying [4]. The second type is long-range (or long-memory) dependent random fields and processes. The literature shows diverse definitions of long-range dependent random fields [4-6]. Most of these definitions are based on the second-order properties of random fields such as asymptotics of the covariance functions at infinity or spectral densities at zero. The most popular definition assumes that a random field is long-range dependent if its covariance function decays hyperbolically. These fields have a power singularity in the spectrum at the origin $[5,7]$.

This thesis studies the asymptotic behaviour of nonlinear functionals of longrange dependent random fields. These functionals play a key role in the theory and statistical applications of random fields [8]. They often produce non-Gaussian

Thesis submitted to La Trobe University in September 2019; degree approved on 11 December 2019; principal supervisor Andriy Olenko, co-supervisor Agus Salim.

(C) 2020 Australian Mathematical Publishing Association Inc. 
asymptotic distributions. The results obtained in this thesis are based on two scenarios. In the first scenario, integral functionals

$$
X_{r_{1}, r_{2}, \kappa}^{(c)}:=d_{r_{1}, r_{2}, \kappa}^{-1} \int_{0}^{r_{1}} \int_{0}^{r_{2}} g(t, s) H_{\kappa}(\xi(t, s)) d t d s
$$

for functional data are considered and the relation between the asymptotics of these functionals and the corresponding additive functionals,

$$
X_{r_{1}, r_{2}, \kappa}^{(d)}:=d_{r_{1}, r_{2}, \kappa}^{-1} \sum_{i=0}^{\left[r_{1}\right]-1} \sum_{j=0}^{\left[r_{2}\right]-1} g(i, j) H_{\kappa}(\xi(i, j)),
$$

is addressed.

The case of the random field $\xi(\cdot, \cdot)$ exhibiting a long-range dependent property and nonrandom functions $g(\cdot, \cdot)$ and $H_{\kappa}(\cdot)$ ( $\kappa$ th Hermite polynomial) is considered. It is shown that these functionals and the corresponding additive functionals have the same asymptotic distribution when $r_{1}$ and $r_{2}$ approach infinity. It is also proved that the analogous results hold true for integral functionals over multidimensional rectangles.

Then we generalise these results to a more general case. More precisely, we investigate the asymptotic behaviour of functionals of the form

$$
Y_{r, K}^{(c)}:=d_{r, K}^{-1} \int_{\Delta_{n}(r)} g(x) H_{\kappa}(\xi(x)) d x,
$$

where $d_{r, K}$ is the normalising factor.

The case of an arbitrary bounded observation window $\Delta_{n} \subset \mathbb{R}^{n}$ is considered. The boundary of $\Delta_{n}$ can have a finite number of discontinuities as functions of $\left(x_{1}, \ldots, x_{n-1}\right)$. The asymptotic behaviour is studied for the increasing domain case, that is, $\Delta_{n}(r)$ is an increasing observation window. The field $\xi(x), x \in \mathbb{R}^{n}$, exhibits the long-range dependent property and the functions $g(\cdot)$ and $H_{K}(\cdot)$ are nonrandom functions. The corresponding additive functionals are defined by

$$
Y_{r, K}^{(d)}:=d_{r, K}^{-1} \sum_{i \in Q_{n}\left(\Delta_{n}(r)\right)} g(\boldsymbol{i}) H_{\kappa}(\xi(\boldsymbol{i})),
$$

where $\boldsymbol{i} \in \mathbb{Z}^{n}$ and $Q_{n}\left(\Delta_{n}(r)\right)$ is a discretised set which corresponds to $\Delta_{n}(r)$.

It is shown that $Y_{r, K}^{(d)}$ and $Y_{r, K}^{(c)}$ have the same non-Gaussian asymptotic distribution when $r$ approaches infinity. This result is applied to derive noncentral limit theorems in both additive and integral functionals cases.

These results generalise the results for the case of one-dimensional processes in [9]. The results are obtained under more general and much weaker assumptions on the weight functions $g(\cdot)$, covariance functions of the fields and spectral densities than those in [9]. Numerical studies are conducted using random fields on squares and observation windows with some jump points. Covariance models such as Cauchy and Bessel covariance functions are used. Also, simulation studies of convergence rates are presented. The numerical studies support the theoretical results and suggest 
various new research problems. We also provide numerical studies for Bessel random fields with oscillating covariance structures. The numerical studies suggest that the theoretical results also hold for this class of random fields.

In the second scenario, functionals of filtered random fields are considered to obtain new limit theorems with non-Gaussian self-similar random processes. The functionals are defined as convolutions of nonrandom kernels with nonlinear functions of random fields. Namely, we investigate functionals of the form

$$
X_{r}(t):=d_{r}^{-1} \int_{\Delta\left(r t^{1 / n}\right)} \int_{\mathbb{R}^{n}} G(\|y-x\|) S(\xi(y)) d y d x, \quad t \in[0,1],
$$

as $r \rightarrow \infty$, where $G(\cdot), S(\cdot)$ are nonrandom functions and $\xi(\cdot)$ is a long-range dependent random field. The case of increasing observation windows $\Delta(r)$ is considered.

It is proved that the asymptotics of $X_{r}(t)$ are non-Gaussian self-similar processes that are given in terms of Wiener-Itô stochastic integrals. The Hurst parameter $H$ of the limit processes is investigated by considering various examples of the set $\Delta \subset \mathbb{R}^{n}$. It is shown that the asymptotic processes have self-similar parameters $H \in(\gamma(\Delta), 1)$ where the lower bound $\gamma(\Delta) \geq 0$ depends on the geometric properties of $\Delta \subset \mathbb{R}^{n}$. It is also demonstrated that in the one-dimensional case $(n=1), \gamma(\Delta)=0$, which coincides with the known one-dimensional results in the literature.

Most published results on this topic give asymptotic random processes that always exhibit nonnegative autocorrelation structures and have the self-similar parameter $H \in\left(\frac{1}{2}, 1\right)$. The limit processes obtained in this thesis can have the self-similar parameter $H \in\left(0, \frac{1}{2}\right)$. These results extend the theory for one-dimensional processes and sequences given in $[5,10,11]$ to multidimensional settings. The results are obtained for random fields under more general assumptions on their spectral densities and covariance functions than those in the aforementioned works.

The main results of the thesis have been published in [1-3].

\section{References}

[1] T. Alodat, N. Leonenko and A. Olenko, 'Limit theorems for filtered long-range dependent random fields', Stochastics (to appear).

[2] T. Alodat and A. Olenko, 'Weak convergence of weighted additive functionals of long-range dependent fields', Theory Probab. Math. Statist. 97 (2017), 9-23.

[3] T. Alodat and A. Olenko, 'Asymptotic behaviour of discretized functionals of long-range dependent functional data', Comm. Statist. Theory Methods (to appear).

[4] J. Beran, Y. Feng, S. Ghosh and R. Kulik, Long-Memory Processes (Springer, Berlin, 2013).

[5] P. Doukhan, G. Oppenheim and M. S. Taqqu, Theory and Applications of Long-Range Dependence (Birkhäuser, Boston, 2002).

[6] A. Ivanov and N. Leonenko, Statistical Analysis of Random Fields (Springer, Dordrecht, 1989).

[7] N. Leonenko and A. Olenko, 'Tauberian and Abelian theorems for long-range dependent random fields', Methodol. Comput. Appl. Probab. 15(4) (2013), 715-742.

[8] N. Leonenko and A. Olenko, 'Sojourn measures of Student and Fisher-Snedecor random fields', Bernoulli 20(3) (2014), 1454-1483.

[9] N. Leonenko and E. Taufer, 'Weak convergence of functionals of stationary long memory processes to Rosenblatt-type distributions', J. Statist. Plann. Inference 136(4) (2006), 1220-1236. 
[10] P. Major, 'Limit theorems for non-linear functionals of Gaussian sequences', Z. Wahrsch. verw. Gebiete 57(1) (1981), 129-158.

[11] M. Rosenblatt, 'Some limit theorems for partial sums of quadratic forms in stationary Gaussian variables', Probab. Theory Related Fields 49(2) (1979), 125-132.

TAREQ ALODAT, Department of Mathematics and Statistics, La Trobe University, Melbourne, Victoria 3086, Australia e-mail: alodat.t@students.latrobe.edu.au 